Annals of Pure and Applied Mathematics

Vol. 15, No. 2, 2017, 243-251

ISSN: 2279-087X (P), 2279-0888(online)

Published on 11 December 2017

www.researchmathsci.org

DOI: http://dx.doi.org/10.22457/apam.v15n2a11

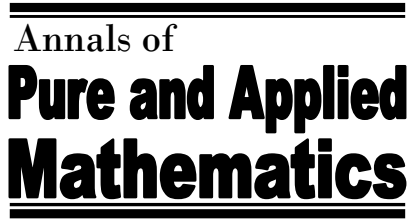

\title{
Fuzzy Implication on the New Representation of Discrete Fuzzy Numbers
}

\author{
Thangaraj Beaula ${ }^{1}$ and Johnson. ${ }^{2}$ \\ ${ }^{1}$ Department of Mathematics, T.B.M.L College, Porayar - 609307, India \\ ${ }^{2}$ Department of Mathematics, DVC College of Education, Srimushnam 608703 \\ E-mail: edwinbeaula@yahoo.co.in
}

Received 18 October 2017; accepted 10 December 2017

Abstract. An implication function $I$ on $u: L \longrightarrow[0,1]$ is defined on the finite chain $L=\{0,1,2, \ldots, \mathrm{n}\}$ a method for extending $I$ to the set of discrete fuzzy numbers whose support is a set of consecutive natural numbers contained in $L$ (denoted by $\mathfrak{F}[L]$ ) is given The resulting extension is in the fact a fuzzy implication on $\mathfrak{F}[L]$ preserving some boundary properties.

Keywords: Fuzzy matrix, complement of fuzzy matrix, sup i-composition, relativity function, medical diagnosis and decision making.

\section{AMS Mathematics Subject Classification (2010): 15B15}

\section{Introduction}

As a generalization of implications in a classical logic, they are used not only to model fuzzy conditionals, but also in the inference process through the modus ponens and tollens rules $[1,8]$. Recently, another approach deals with the possibility of extending monotonic operations on $L$ to operations on the set discrete fuzzy numbers whose support is asset of consecutive natural numbers contained in $L$. More specifically, the concept of discrete fuzzy number was introduced in[13] as a fuzzy subset of $\mathbb{R}$ with discrete support and analogous properties to a fuzzy number.

It is well known that arithmetic and lattice operations between fuzzy numbers are defined using the Zadeh's extension principle [7]. In particular, it is proved in [4] the set $\mathfrak{F}[L]$ of discrete fuzzy numbers whose support is a set of consecutive natural numbers contained in $L$, is a distributive lattice. Following with this idea to study this paper the possibility of extending discrete implications on $L$ to the implications defined on [7]. Moreover some boundary properties on fuzzy implications are preserved under this extension.

\section{Preliminaries}

\subsection{Discrete implications}

Let $(P, \leq)$ be a bounded ordered set with smallest element 0 and greatest element 1 .

Definition 2.2. An implication function $I$ on $(P, \leq)$ is a binary operator $I: P \times P \rightarrow P$ that is decreasing in the first variable, increasing in the second one and satisfies the conditions $I(0,0)=1, I(1,1)=1$ and $I(1,0)=0$. 


\section{Thangaraj Beaula and Johnson B}

Definition 2.4. [6] A border implication $I$ on $(P, \leq)$ is a implication function that satisfies the neutrality principle $I(1, \beta)=\beta$ for all $\beta \in P$

Definition 2.5. Let $I$ be an implication function on $(P, \leq)$, then $I$ satisfies

(i) The exchange principle (EP), if $I(x, I(y, z))=I(y, I(x, z))$ for all $x, y, z \in P$

(ii) The contraposition law (CL) with respect to the strong negation $\mathrm{N}$ on $P$, if $I(N(y), N(x))=I(x, y)$ for all $x, y \in P$

(iii) The ordering property (OP), if $I(x, y)=1 \Leftrightarrow x \leq y$ for all $x, y \in P$.

A particular case of bounded ordered set is when consider the finite chain $L=\{0,1, \ldots, \mathrm{n}\} \subset \mathrm{N}$. Operations defined on $L$ are usually called discrete operations and they have been studied by many authors(see[6,9,11]) In these studies the following condition, generally used as discrete counterpart of continuity, is usually considered.

\subsection{Discrete fuzzy number}

In this section, we recall some definitions and main results about discrete fuzzy numbers which will be used later. By a fuzzy subset of $\mathbb{R}$, we mean a function $A: \mathbb{R} \rightarrow[0,1]$ For each fuzzy subset $A$,

Let ${ }^{\alpha} A=\{x \in \mathbb{R}: A(x) \geq \alpha\}$ for any $\alpha \in(0,1]$ be its $\alpha$-cut by supp(A)

Definition 2.7. A fuzzy subset A of $\mathbb{R}$ with membership mapping $A: \mathbb{R} \rightarrow[0,1]$ is called

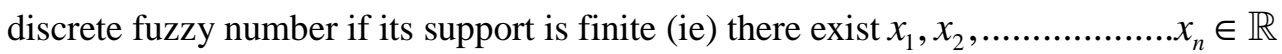
with $x_{1}<x_{2}<\ldots<x_{n}$ such that $\operatorname{supp}(\mathrm{A})=\left\{x_{1}, x_{2}, \ldots \ldots \ldots \ldots \ldots \ldots x_{n}\right\}$ and there are natural numbers $s, t$ with $1 \leq s \leq t \leq n$ such that

(i) $u\left(x_{i}\right)=1$ for any natural number $i$ with $s \leq i \leq t$

(ii) $u\left(x_{i}\right) \leq u\left(x_{j}\right)$ for each natural numbers $i, j$ with $1 \leq i \leq j \leq s$

(iii) $u\left(x_{i}\right) \geq u\left(x_{j}\right)$ for each natural number $i, j$ with $t \leq i \leq j \leq n$.

Remark 2.8. If the fuzzy subset A is a discrete fuzzy number then the support of A co insides with its closure(ie) $\operatorname{supp}(\mathrm{A})=\mathrm{A}^{0}$. From now on, we will denote the set of discrete fuzzy numbers by DFN and the abbreviation $\boldsymbol{d} \boldsymbol{f} \boldsymbol{n}$ will denote a discrete fuzzy numbers.

Theorem 2.9. [14] (Representation of discrete fuzzy number) Let A be a discrete fuzzy number. Then following statements (1)-(4) hold.

i) ${ }^{\alpha} A$ is a non empty finite subset of , $\mathbb{R}$ for any $\alpha \in(0,1]$

ii) ${ }^{\alpha_{2}} A \subseteq^{\alpha_{1}} A$ for any $\alpha_{1}, \alpha_{2} \in(0,1]_{\text {with }} 0 \leq \alpha_{1} \leq \alpha_{2} \leq 1$

iii) For any $\alpha_{1}, \alpha_{2} \in(0,1]$ with $0 \leq \alpha_{1} \leq \alpha_{2} \leq 1$ if $x \in{ }^{\alpha_{1}} A-{ }^{\alpha_{2}} A$ we have $x<y$ for all $y \in \in^{\alpha_{2}} A$ or $x>y$ for all $y \in \in^{\alpha_{2}} A$ 
Fuzzy Implication on the New Representation of Discrete Fuzzy Numbers

iv) For any $\alpha_{0} \in(0,1]$ there exist some real numbers $\alpha_{0}^{\prime}$ with $0<\alpha_{0}^{\prime}{ }^{\prime} \alpha_{0}$ such that ${ }^{\alpha_{0}{ }^{\prime}} A={ }^{\alpha_{0}} A$ (ie) ${ }^{\alpha} A={ }^{\alpha_{0}} A$ for any $\alpha \in\left[\alpha_{0}{ }^{\prime}, \alpha_{0}\right]$

Theorem 2.10. [14] Conversely, if for any $\alpha \in[0,1]$ there exists ${ }^{\alpha} A \in \mathbb{R}$ satisfying analogous condition to the (1)-(4) of theorem 2.10, then there exists a unique $A \in D F N$ such that its $\alpha$-cuts are exactly the sets ${ }^{\alpha} A$ for any $\alpha \in[0,1]$

\subsection{Maximum and minimum of discrete fuzzy numbers}

Let $\mathrm{u}, \mathrm{v}$ be two dfn and ${ }^{\alpha} u=\left\{{ }^{\alpha} x_{1}, \ldots \ldots \ldots \ldots . .{ }^{\alpha} x_{p}\right\}$ and ${ }^{\alpha} v=\left\{{ }^{\alpha} y\right.$ .$\left.^{\alpha} y_{k}\right\}$ their $\alpha$-cuts

respectively. For each $\alpha \in[0,1]$, we consider the following sets

$\min ^{\alpha}(u, v)=\left\{z \in \operatorname{supp}(\mathrm{u}) \wedge \operatorname{supp}(\mathrm{v}) \operatorname{such}\right.$ that $\left.\min \left({ }^{\alpha} x_{1},{ }^{\alpha} y_{1}\right) \leq z \leq \min \left({ }^{\alpha} x_{p},{ }^{\alpha} y_{k}\right)\right\}$

and

$\max ^{\alpha}(u, v)=\left\{z \in \operatorname{supp}(\mathrm{u}) \vee \operatorname{supp}(\mathrm{v}) \operatorname{such}\right.$ that $\left.\max \left({ }^{\alpha} x_{1},{ }^{\alpha} y_{1}\right) \leq z \leq \max \left({ }^{\alpha} x_{p},{ }^{\alpha} y_{k}\right)\right\}$

where $\operatorname{supp}(\mathrm{u}) \wedge \operatorname{supp}(\mathrm{v})=\{z=\min (x, y) / x \in \operatorname{supp}(u), \mathrm{y} \in \operatorname{supp}(\mathrm{v})\}$

and $\operatorname{supp}(\mathrm{u}) \vee \operatorname{supp}(\mathrm{v})=\{z=\max (x, y) / x \in \operatorname{supp}(u), \mathrm{y} \in \operatorname{supp}(\mathrm{v})\}$

Proposition 2.12. [3] There exists two unique discrete fuzzy numbers, that we will denote by $\operatorname{MIN}(u, v)$ an $\operatorname{MAX}(u, v)$, such that they have the sets $\min ^{\alpha}(u, v)$ and $\max ^{\alpha}(u, v)$ as $\alpha$-cuts respectively.

The following result holds for $[L]$ but is not true for the set of discrete fuzzy numbers in general (See[4]).

Theorem 2.13. [4] The triplet ( [L],MIN,MAX )is a bounded distributive lattice where $\mathrm{N} \in \mathfrak{F}[L]$ (the unique discrete fuzzy number whose support is the singleton $\{\mathrm{n}\}$ ) and $0 \in \mathfrak{F}[L]$ (the unique discrete fuzzy number whose support is a singleton $\{0\}$ ) are the maximum and minimum respectively.

Remark 2.14. [4] Using these operations, define a partial order on $\{[L]$ in the usual way: $u \leqslant v$ if and only if $\operatorname{MIN}(u, v)=u$, or equivalently, $u \leqslant v$ if and only if $M A X(u, v)=v$ for any $u, v \in \mathfrak{F}[\mathrm{L}]$. Equivalently, also define the partial ordering in terms of $\alpha$-cuts

$\mathrm{u} \preccurlyeq \mathrm{v}$ if and only if $\left({ }^{\alpha} u,{ }^{\alpha} v\right)={ }^{\alpha} u$

$\mathrm{u} \preccurlyeq \mathrm{v}$ if and only if $\left({ }^{\alpha} u,{ }^{\alpha} v\right)={ }^{\alpha} v$

\section{Implication functions on $[L]$}

Implication function on the bounded set $[L]$ constructed from a discrete implication function $I$ defined on a the discrete finite chain $L$.

If $O: L \times L \rightarrow L$

$(x, y) \rightarrow O(x, y)$ is a binary discrete function on $L$, denoted by $\mathrm{O}$, the binary operation

$O: 2^{L} \times 2^{L} \rightarrow 2^{L}(X, Y) \rightarrow O(X, Y)$ where $O(X, Y)=\{O(x, y) / x \in X, y \in Y\}$ 


\section{Thangaraj Beaula and Johnson B}

\subsection{Notation}

For $A \subset R$ denote $\max A=\max \{x: x \in A\}$, and $\min A=\min \{x: x \in A\}$. Let $u$ be a fuzzy set of $R$, and $[u]^{0}$ be finite,$u=[\underline{u}(r), \bar{u}(r)]$

$$
\begin{aligned}
& { }^{\alpha} \underline{u}(r)=\left\{x \in[u]^{0} / \underline{u}(r) \leq \alpha\right\} \\
& { }^{\alpha} \bar{u}(r)=\left\{x \in[u]^{0} / \bar{u}(r) \geq \alpha\right\} \text { with } \alpha \in[0,1] \text { and } r \in A \\
& \text { Let }^{\alpha} u=\left[\left({ }^{\alpha} \underline{u}(r) \cup \cup^{\alpha} \bar{u}(r)\right)\right],{ }^{\alpha} v=\left[\left({ }^{\alpha} \underline{v}(r) \cup \cup^{\alpha} \bar{v}(r)\right)\right] \\
& \text { Define } \min I\left({ }^{\alpha} \underline{u}(r),{ }^{\alpha} \underline{v}(r)\right)=I\left(\max ^{\alpha} \underline{u}(r), \min ^{\alpha} \underline{v}(r)\right) \\
& \min I\left({ }^{\alpha} \bar{u}(r),{ }^{\alpha} \bar{v}(r)\right)=I\left(\max ^{\alpha} \bar{u}(r), \min ^{\alpha} \bar{u}(r)\right) \\
& \max I\left({ }^{\alpha} \underline{u}(r),{ }^{\alpha} \underline{v}(r)\right)=I\left(\min ^{\alpha} \underline{u}(r), \max ^{\alpha} \underline{v}(r)\right) \\
& \max I\left({ }^{\alpha} \bar{u}(r),{ }^{\alpha} \bar{v}(r)\right)=I\left(\min ^{\alpha} \bar{u}(r), \max ^{\alpha} \bar{u}(r)\right)
\end{aligned}
$$

Lemma 3.2. Let us consider $u, v \in \mathfrak{F}[L]$ and let $I$ be a discrete implication function on the finite chain $L$. Then the following results hold.

$$
\begin{aligned}
& \min I\left({ }^{\alpha} \underline{u}(r),{ }^{\alpha}{ }^{\alpha} \frac{v}{\bar{v}}(r)\right)=I\left(\max ^{\alpha} \underline{\underline{u}}(r), \min ^{\alpha} \underline{\underline{v}}(r)\right) \\
& \min I\left({ }^{\alpha} \bar{u}(r),{ }^{\alpha}(r)\right)=I\left(\max ^{\alpha} \bar{u}(r), \min ^{\alpha} \bar{v}(r)\right) \\
& \max I\left({ }^{\alpha} \underline{u}(r),{ }^{\alpha} \underline{v}(r)\right)=I\left(\min ^{\alpha} \underline{\underline{u}}(r), \max ^{\alpha}{ }^{\alpha} \frac{\underline{v}}{-}(r)\right) \\
& \max I\left({ }^{\alpha} \bar{u}(r),{ }^{\alpha} \bar{v}(r)\right)=I\left(\min ^{\alpha} \frac{u}{u}(r), \max ^{\alpha} \frac{v}{v}(r)\right)
\end{aligned}
$$

It is obvious that

$$
I\left(\min ^{\alpha} \underline{u}(r), \max ^{\alpha} \underline{v}(r)\right) \leq \max I\left({ }^{\alpha} \underline{u}(r),{ }^{\alpha} \underline{v}(r)\right) \text { is clear }
$$

To prove the converse inequality. Since $I$ is decreasing in the first variable and increasing in the second one we get

$$
I(x, y) \leq I\left(\min ^{\alpha} \underline{u}(r), \max ^{\alpha} \underline{v}(r)\right) \text { for all } x \in^{\alpha} \underline{u}(r) \text { and for all } y \in^{\alpha} \underline{v}(r)
$$

Thus max $I\left({ }^{\alpha} \underline{u}(r),{ }^{\alpha} \underline{v}(r)\right) \leq I\left(\min ^{\alpha} \underline{u}(r), \max ^{\alpha} \underline{v}(r)\right)$

Similarly $I(x, y) \leq I\left(\min ^{\alpha} \bar{u}(r), \max ^{\alpha} \bar{v}(r)\right)$ for all $x \in^{\alpha} \bar{u}(r)$ and for all $y \epsilon^{\alpha} \bar{v}(r)$

$\Rightarrow \max I\left({ }^{\alpha} \bar{u}(r),{ }^{\alpha} \bar{v}(r)\right) \leq I\left(\min ^{\alpha} \bar{u}(r), \max ^{\alpha} \bar{v}(r)\right)$

and $I(x, y) \leq I\left(\max ^{\alpha} \underline{u}(r), \min ^{\alpha} \underline{v}(r)\right)$ for all $x \in \in^{\alpha} \underline{u}(r)$ and for all $y \in^{\alpha} \underline{v}(r)$

$\Rightarrow \min I\left({ }^{\alpha} \underline{u}(r),{ }^{\alpha} \underline{v}(r)\right) \leq I\left(\max ^{\alpha} \underline{u}(r), \min ^{\alpha} \underline{v}(r)\right)$

And $I(x, y) \leq I\left(\max ^{\alpha} \bar{u}(r), \min ^{\alpha} \bar{v}(r)\right)$ for all $x \in^{\alpha} \bar{u}(r)$ and for all $y \in^{\alpha} \bar{v}(r)$

$$
\Rightarrow \min I\left(\alpha_{u(r)}^{-},{ }^{-}{ }_{v(r))}^{-} \leq I\left(\max { }_{u}^{-}(r), \min _{v(r))} \alpha_{v}^{-}\right.\right.
$$

Proposition 3.3. Let us consider $u, v \in \mathfrak{F}[L]$ and let $I$ be a discrete implication function on the finite chain $L$. There exists a unique discrete fuzzy number whose $\alpha$-cuts are the exactly the sets

$$
\alpha_{C(r)}=\left\{z \in L / \min I\left[{ }^{\alpha}{ }^{u(r),}, \alpha_{\underline{v}(r)] \leq z \leq \max I[} \alpha_{\underline{u}(r),} \alpha_{\underline{v}(r)]}\right.\right.
$$

Denoted by $\mathfrak{I}(\mathrm{u}, \mathrm{v})$ moreover $\mathfrak{I}(\mathrm{u}, \mathrm{v}) \in[L]$. 
Fuzzy Implication on the New Representation of Discrete Fuzzy Numbers

Proof: To prove that the sets ${ }^{\alpha} C_{(r)}$ satisfy the four conditions of the Wange's theorem (theorem 2.12 and 2.13)

(i) For each $\alpha \in[0,1],{ }^{\alpha} C_{(r)}$ is a non empty finite set ,because of ${ }^{\alpha} u$ and ${ }^{\alpha} v$ are both non empty finite sets(the discrete fuzzy numbers are normal fuzzy subsets)

(ii) ${ }^{\beta} C_{(r)} \subseteq{ }^{\alpha} C_{(r)}$ for any $\alpha, \beta \in[0,1]$ with $0 \leq \alpha \leq \beta \leq 1$ Because if $\mathrm{u}, \mathrm{v} \in \mathfrak{F}[L]$ then

${ }^{\beta} u \subseteq{ }^{\alpha} u$ implies $\min \left({ }^{\alpha} \underline{u}(r)\right) \leq \min \left({ }^{\beta} \underline{u}(r)\right)$

$\min \left({ }^{\alpha} \bar{u}(r)\right) \leq \min \left({ }^{\beta} \bar{u}(r)\right)$

And $\max \left({ }^{\alpha} \underline{u}(r)\right) \leq \max \left({ }^{\beta} \underline{u}(r)\right)$

$\max \left({ }^{\alpha} \bar{u}(r)\right) \leq \max \left({ }^{\beta} \bar{u}(r)\right)$

$\min \left({ }^{\alpha} \bar{v}(r)\right) \leq \min \left({ }^{\beta} \bar{v}(r)\right)$

$\max \left({ }^{\alpha} \bar{v}(r)\right) \leq \max \left({ }^{\beta} \bar{v}(r)\right)$

Moreover, as I is decreasing in the first variable using relation(3),(4) to get

$I(\max \alpha \underline{u}(r), z) \leq I\left(\max \beta_{\underline{u}}(r), z\right)$

$I(\max \alpha \bar{u}(r), z) \leq I(\max \beta \bar{u}(r), z)$ for all $z \in L$

By relation(5),(6)

$I\left[\max \alpha \underline{u}(r), \min ^{\alpha} \alpha_{\underline{v}(r)] \leq I[\max } \beta_{\underline{u}(r), \min } \beta_{\underline{v}(r)]}\right.$

$I[\max \alpha \bar{u}(r), \min \alpha \bar{v}(r)] \leq I[\max \beta \bar{u}(r), \min \beta \bar{v}(r)]$

Thus, from lemma(3.2)

$\min I\left[{ }^{\alpha}{ }_{u(r)}{ }^{\alpha}{ }_{\underline{v}(r)] \leq \min I[} \beta_{\underline{u}(r),} \beta_{\underline{v}(r)]}\right.$

$\min I\left[\alpha_{u(r),}^{-}{ }^{-}{ }_{v(r)] \leq \min I[}^{\beta-} \bar{u}_{(r),} \beta_{\bar{v}(r)]}\right.$

Similarly

$\max I\left[{ }^{\alpha}{ }_{\underline{u}(r),}{ }^{\alpha}{ }_{\underline{v}(r)] \leq \max I[} \beta_{\underline{u}(r),}{ }^{\beta} \underline{\underline{v}(r)]}\right.$

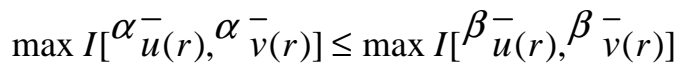

Comparing (11),(12)and(13),(14)

${ }^{\beta} C=\left\{z \in L / \min I\left[{ }^{\beta} \underline{u}(r),{ }^{\beta} \underline{v}(r)\right] \leq \mathrm{z} \leq \max I\left[{ }^{\beta}{ }_{\underline{u}(r),}{ }^{\beta} \underline{v}(r)\right]\right.$,

$\left.\min I\left[{ }_{\bar{u}(r),} \beta \bar{v}(r)\right] \leq \mathrm{z} \leq \max I\left[{ }^{\beta} \bar{u}(r),{ }^{\beta} \bar{v}(r)\right]\right\} \subseteq\left\{z \in L / \min I{ }^{\alpha} \underline{u}(r),{ }^{\alpha} \underline{v}(r)\right] \leq \mathrm{z} \leq$

$\max I\left[^{\alpha} \underline{u}(r),{ }^{\alpha} \underline{v}(r)\right]$,

$\left.\min I\left[{ }^{\alpha} \bar{u}(r),{ }^{\alpha} \bar{v}(r)\right] \leq \mathrm{z} \leq \max I\left[{ }^{\alpha} \bar{u}(r),{ }^{\alpha} \bar{v}(r)\right]\right\}={ }^{\alpha} C_{(r)}$

Therefore ${ }^{\beta} C_{(r)} \subseteq{ }^{\alpha} C_{(r)}$

(i) If $x \in{ }^{\beta} C_{(r)}-{ }^{\alpha} C_{(r)}$ then $x \in L$ and $x$ does not belong to ${ }^{\beta} C_{(r)}$ hence either

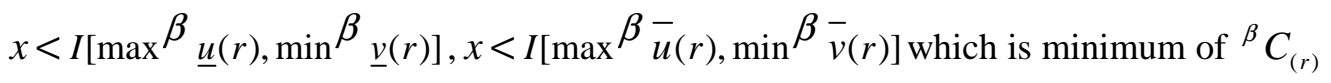
(or) 
Thangaraj Beaula and Johnson B

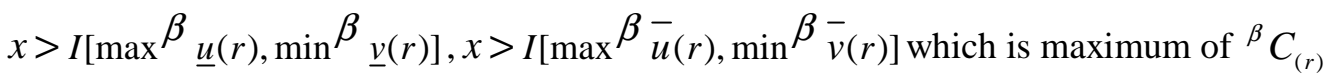

(ii) As u,veF[L], according to theorem 2.11for each $\alpha \in[0,1]$ there exists real numbers

$\alpha_{1}^{\prime}$ and $\alpha_{2}^{\prime}$ with $0<\alpha_{1}^{\prime} \alpha_{\text {and } 0<} \alpha_{2}^{\prime}{ }_{<} \alpha_{\text {such that for each }} \alpha \in\left[\alpha_{1}^{\prime}, \alpha\right]$

${ }^{\alpha} \underline{u}(r)={ }^{r} \underline{u}(r)$ and $\quad \alpha \bar{u}(r)={ }^{r} \bar{u}(r)$ moreover

$$
{ }^{\alpha} \underline{v}(r)={ }^{r} \underline{v}(r) \text { and }{ }^{\alpha} \bar{v}(r)={ }^{r} \bar{v}(r) \text { for each } \alpha \in\left[\alpha_{2}^{\prime}, \alpha\right]
$$

Thus if $\alpha^{\prime}=\alpha_{1}^{\prime} \vee \alpha_{2}^{\prime}$ to get

$\min \left({ }^{r} \underline{u}(r)\right)=\min \left({ }^{\alpha} \underline{u}(r)\right)$ and $\max \left({ }^{r} \underline{u}(r)=\max \left({ }^{\alpha} \underline{u}(r)\right)\right.$

$\min \left({ }^{r} \bar{u}(r)\right)=\min \left({ }^{\alpha} \bar{u}(r)\right)$ and $\max \left({ }^{r} \bar{u}(r)=\max \left({ }^{\alpha} \bar{u}(r)\right)\right.$

$\min \left({ }^{r} \underline{v}(r)\right)=\min \left({ }^{\alpha} \underline{v}(r)\right)$ and $\max \left({ }^{r} \underline{v}(r)=\max \left({ }^{\alpha} \underline{v}(r)\right)\right.$

$\min \left({ }^{r} \bar{v}(r)\right)=\min \left({ }^{\alpha} \bar{v}(r)\right)$ and $\max \left({ }^{r} \bar{v}(r)=\max \left({ }^{\alpha} \bar{v}(r)\right)\right.$ for each $\alpha \in\left[\alpha^{\prime}, \alpha\right]$

$I\left[\max ^{r} \underline{u}(r), \min ^{r} \underline{v}(r)\right]=I\left[\max ^{\alpha} \underline{u}(r), \min ^{\alpha} \underline{v}(r)\right]$

$I\left[\max ^{r} \bar{u}(r), \min ^{r} \bar{v}(r)\right]=I\left[\max ^{\alpha} \bar{u}(r), \min ^{\alpha} \bar{v}(r)\right]$

$I\left[\min ^{r} \underline{u}(r), \max ^{r} \underline{v}(r)\right]=I\left[\min ^{\alpha} \underline{u}(r), \max ^{\alpha} \underline{v}(r)\right]$

Hence by lemma 3.1,

$\min I\left[^{r} \underline{u}(r),{ }^{r} \underline{v}(r)\right]=\min I\left[{ }^{\alpha} \underline{u}(r),{ }^{\alpha} \underline{v}(r)\right]$
$\min I\left[^{r} \bar{u}(r),{ }^{r} v(r)\right]=\min I\left[{ }^{\alpha} \bar{u}(r),{ }^{\alpha} \bar{v}(r)\right]$
$\max I\left[^{r} \underline{u}(r),{ }^{r} \underline{v}(r)\right]=\max I\left[{ }^{\alpha} \underline{u}(r),{ }^{\alpha} \underline{v}(r)\right]$
$\max I\left[{ }^{r} \bar{u}(r),{ }^{r} v(r)\right]=\max I\left[{ }^{\alpha} \bar{u}(r),{ }^{\alpha} \bar{v}(r)\right]$

And so

${ }^{r} C=\left\{z \in L / \min I\left[{ }^{r} \underline{u}(r),{ }^{r} \underline{v}(r)\right] \leq \mathrm{z} \leq \max I\left[^{r} \underline{u}(r),{ }^{r} \underline{v}(r)\right]\right.$,

$\left.\min I\left[{ }^{r} \bar{u}(r),{ }^{r} \bar{v}(r)\right] \leq \mathrm{z} \leq \max I\left[^{r} \bar{u}(r),{ }^{r} \bar{v}(r)\right]\right\}=\left\{z \in L / \min I\left[{ }^{\alpha} \underline{u}(r),{ }^{\alpha} \underline{v}(r)\right] \leq \mathrm{z} \leq\right.$

$\max I\left[{ }^{\alpha} \underline{u}(r),{ }^{\alpha} \underline{v}(r)\right]$,

$\left.\min I\left[{ }^{\alpha} \bar{u}(r),{ }^{\alpha} \bar{v}(r)\right] \leq \mathrm{z} \leq \max I\left[{ }^{\alpha} \bar{u}(r),{ }^{\alpha} \bar{v}(r)\right]\right\}={ }^{\alpha} C$ for each $\alpha \in\left[\alpha^{\prime}, \alpha\right]$

As the $\operatorname{sets}^{\alpha} C$ fulfill for each $\alpha \in[0,1]$ the conditions stated in theorem 2.11 by theorem 2.12 there exits a unique discrete number, that will be denoted by $I(\mathrm{u}, \mathrm{v})$ such that its $\alpha$-cuts are exactly these sets.

Further, from the construction of the sets ${ }^{\alpha} C$ for each $\alpha \in[0,1]$ it is straight forward to see that are sets of consecutive natural numbers belonging to the finite chain $L$. Thus $I(\mathrm{u}, \mathrm{v}) \in[L]$.

The previous proposition will allow us to define a binary operation $I$ on $\mathfrak{F}[L]$ from an implication function $I$ defined on the finite chain $L$. 
Fuzzy Implication on the New Representation of Discrete Fuzzy Numbers

Definition 3.4. Let us consider an implication function $I$ on the finite chain $L$. The binary operation on $[L]$ defined as follows $I:[L] \times \mathfrak{F}[L] \longrightarrow \mathfrak{F}[L]$

$(u, v) \mapsto I(u, v)$ will be called the extension of the discrete implication I to $\mathfrak{F}[L]$, being $I(\mathrm{u}, \mathrm{v})$ the discrete fuzzy number whose $\alpha$-cuts are the sets

$\left\{z \in L / \min I\left[{ }^{\alpha} \underline{u}(r),{ }^{\alpha} \underline{v}(r)\right] \leq \mathrm{z} \leq \max I\left[{ }^{\alpha} \underline{u}(r),{ }^{\alpha} \underline{v}(r)\right]\right.$,

$\left.\min I\left[{ }^{\alpha} \bar{u}(r),{ }^{\alpha} \bar{v}(r)\right] \leq \mathrm{z} \leq \max I\left[{ }^{\alpha} \bar{u}(r),{ }^{\alpha} \bar{v}(r)\right]\right\}$ for each

Theorem 3.5. Let $I$ be an implication function on $L$. Then the extension of the discrete implication function $I, I$ is an implication function on $\mathfrak{F}[L]$.

Proof: To show this result, prove all the conditions stated in definition 2.1.Let us consider the $u, v \in \mathfrak{F}[L]$.To see that $I$ is decreasing in the first variable,(ie) if $\mathrm{u} \preccurlyeq \mathrm{v}$ then $\mathfrak{I}$ $(\mathrm{u}, \mathrm{w}) \geqslant \mathfrak{T}(\mathrm{v}, \mathrm{w})$ for all $\mathrm{w} \in \mathfrak{F}[L]$.

According to remark 2.13 is equivalent to prove that $\min \left(\mathfrak{T}^{\alpha}(v, w), \mathfrak{I}^{\alpha}(u, w)\right)=\mathfrak{T}$

${ }^{\alpha}(v, w)$ for all $\alpha \in[0,1]$

As $\mathrm{u} \preccurlyeq \mathrm{v}$ then $\min ^{\alpha} \underline{u}(r) \leq \min ^{\alpha} \underline{v}(r), \min ^{\alpha} \bar{u}(r) \leq \min ^{\alpha} \bar{v}(r)$ and

$\max ^{\alpha} \underline{u}(r) \leq \max ^{\alpha} \underline{v}(r)$

$\max ^{\alpha} \bar{u}(r) \leq \max ^{\alpha} \bar{v}(r)$

Thus as $I$ is an implication function on $L$. it is a decreasing function in the first variable then by Lemma3.2.

$\min I\left[{ }^{\alpha} \underline{u}(r),{ }^{\alpha} \underline{w}(r)\right]=I\left[\max ^{\alpha} \underline{u}(r), \min ^{\alpha} \underline{w}(r)\right]$

$\geq\left[\max ^{\alpha} \underline{v}(r), \min ^{\alpha} \underline{w}(r)\right]$

$$
=\min I\left[^{\alpha} \underline{v}(r),^{\alpha} \underline{w}(r)\right]
$$

Analogously, $\max I\left[{ }^{\alpha} \underline{u}(r),{ }^{\alpha} \underline{w}(r)\right]=I\left[\min ^{\alpha} \underline{u}(r), \max ^{\alpha} \underline{w}(r)\right]$

$\geq\left[\min ^{\alpha} \underline{v}(r), \max ^{\alpha} \underline{w}(r)\right]$

$=\max I\left[{ }^{\alpha} \underline{v}(r),^{\alpha} \underline{w}(r)\right]$

Finally using the relations (15) and (16)

$I^{\alpha}(\underline{u}(r), \underline{w}(r))=\left\{z \in L / \min I\left({ }^{\alpha} \underline{u}(r),{ }^{\alpha} \underline{w}(r)\right) \leq z \leq \max I\left({ }^{\alpha} \underline{u}(r),{ }^{\alpha} \underline{w}(r)\right)\right\}$

$\geq\left\{z \in L / \min I\left({ }^{\alpha} \underline{v}(r),{ }^{\alpha} \underline{w}(r)\right) \leq z \leq \max I\left({ }^{\alpha} \underline{v}(r),{ }^{\alpha} \underline{w}(r)\right)\right\}$

$=I^{\alpha}(\underline{v}(r), \underline{w}(r))$ for each $\alpha \in[0,1]$ The increasing ness with the second variable follows similarly.

With respect to the boundary conditions

$I^{\alpha}(\mathrm{O}(r), \mathrm{O}(r))=\left\{z \in L / \min I\left({ }^{\alpha} \mathrm{O}(r),{ }^{\alpha} \mathrm{O}(r)\right) \leq z \leq \max I\left({ }^{\alpha} \mathrm{O}(r),{ }^{\alpha} \mathrm{O}(r)\right)\right\}$

$=\{\mathrm{n}\}={ }^{\alpha} N$

Because of $\min I\left({ }^{\alpha} \mathrm{O}(r),{ }^{\alpha} \mathrm{O}(r)\right)=\max I\left({ }^{\alpha} \mathrm{O}(r),{ }^{\alpha} \mathrm{O}(r)\right)=\mathrm{n}$ for each $\alpha \in[0,1]$

Then $I(\mathrm{O}(r), \mathrm{O}(r))=N$

The other two conditions $I(\mathrm{~N}, \mathrm{~N})=N$ and $I(\mathrm{~N}, \mathrm{O})=0$ follows similarly. 


\section{Thangaraj Beaula and Johnson B}

Example 3.6. Let us consider the chin $L_{6}$ and $A=\left\{\frac{0.3}{0}, \frac{0.5}{1}, \frac{1}{2}, \frac{0.8}{3}, \frac{0.5}{4}\right\}$,

$$
\begin{aligned}
& B=\left\{\frac{0.6}{2}, \frac{0.8}{3}, \frac{0.9}{4}, \frac{1}{5}, \frac{0.8}{6}\right\} \text { belonging to }\left[L_{6}\right] \text { consider the function } \\
& I(x, y)=\left\{\begin{array}{l}
y-x \text { if } y \geq x \\
0 \quad \text { if } x>y
\end{array}\right. \\
& { }^{0.3} \underline{u}=\{0\}, \quad{ }^{0.3} \bar{u}=\{1,2,3,4\},{ }^{0.3} \underline{v}=\{\}, \\
& { }^{0.5} \underline{u}=\{0,1,4\}, \quad{ }^{0.5} \bar{u}=\{2,3\}, \quad{ }^{0.5} \underline{v}=\{\}, \\
& { }^{0.6} \underline{u}=\{0,1,4\}, \quad{ }^{0.6} \bar{u}=\{2,3\}, \quad{ }^{0.6} \underline{v}=\{2\}, \\
& { }^{0.8} \underline{u}=\{0,1,3,4\} \quad{ }^{0.8} \bar{u}=\{2\} \quad{ }^{0.8} \underline{v}=\{2,3,6\} \\
& { }^{0.9} \underline{u}=\{0,1,3,4\}, \quad{ }^{0.9} \bar{u}=\{2\}, \quad{ }^{0.9} \underline{v}=\{2,3,6\}, \\
& { }^{1} \underline{u}=\{0,1,2,3,4\},{ }^{1} \bar{u}=\{\}, \quad \underline{v}=\{2,3,4,5,6\}, \\
& { }^{0.3} \bar{v}=\{2,3,4,5,6\} \text {, } \\
& { }^{0.5} \bar{v}=\{2,3,4,5,6\} \text {, } \\
& { }^{0.6} \bar{v}=\{3,4,5,6\} \text {, } \\
& 0 . \bar{v}^{-}=\{4,5\} \\
& 0 .{ }^{-} \bar{v}=\{5\}, \\
& { }^{1} \bar{u}=\{\}, \\
& I^{\alpha}(\underline{u}(r), \underline{v}(r))=\left\{z \in L / \min I\left({ }^{\alpha} \underline{u}(r),{ }^{\alpha} \underline{v}(r)\right) \leq z \leq \max I\left({ }^{\alpha} \underline{u}(r),{ }^{\alpha} \underline{v}(r)\right)\right\} \\
& I^{0.3}(\underline{u}(r), v(r))=0 I^{0.6}(\underline{u}(r), \underline{v}(r))=1 \leq z \leq 2 \\
& I^{0.5}(\underline{u}(r), \underline{v}(r))=0,1,4 I^{0.8}(\underline{u}(r), \underline{v}(r))=0 \leq z \leq 3 \& 5 \leq z \leq 6 \\
& I^{1}(\underline{u}(r), \underline{v}(r))=0 \leq z \leq 4 I^{0.9}(\underline{u}(r), \underline{v}(r))=0 \leq z \leq 6
\end{aligned}
$$

\section{REFERENCES}

1. M.Baczynski and B.Jayaram, Fuzzy Implications, Studies in Fuzziness and Soft Computing, 231, Springer, Berlin Heidelberg, 2008.

2. J.Casasnovas and J.V.Riera, On the addition of discrete fuzzy numbers, WSEAS Transactions on Mathematics, 5(5) (2006) 549-554.

3. J.Casasnovas and J.V.Riera, Maximum and minimum of discrete fuzzy numbers, In Frontiers in Artificial Intelligence and Applications; artificial intelligence research and development, 163 (2007) 273-280.

4. J.Casasnovas and J.V.Riera, Lattice properties of discrete fuzzy numbers under extended min and max, In Proceedings IFSA-EUSFLAT, 647-652, July, Lisbon (Portugal), 2009. 
Fuzzy Implication on the New Representation of Discrete Fuzzy Numbers

5. J.Casasnovas and J.V.Riera, Weighted means of subsective Evaluations, In R.Seising, V.Sanz (editors), Soft Computing Inhumanities and Social Sciences, Springer-Verlag, Berlin, Forthcoming.

6. B.DeBaets, Model implications and their characterization, In N. Sreele, editor, proccedings of the First ICSC International Symposium on Fuzzy logic, (ICSC1995), pages A42-A49, Acadamic Press, 1995.

7. G.J.Klir and B.Yuan, Fuzzy sets and fuzzy logic (Theory and Applications), Prentice Hall, 1995.

8. M.Mas, M.Monserrat, J.Torrens and E.Trillas, A survey on Fuzzy Implications Functions, IEEE Transactions on Fuzzy Systems, 15 (2007) 1107-1121.

9. M.Mas, M.Monserrat and J.Torrens, S-implications and R-implications on a finite chain, Kybernetica, 40 (2004) 3-20.

10. M.Mas, M.Monserrat and J.Torrens, On two types of discrete implications, International Journal of Approximate Reasoning, 40 (2005) 262-279.

11. G.Mayor and J.Torrens, Triangular norms on discrete settings, In E.P. Klement and R.Mesiar, editors, Logical, algebraic, Analytic and Probabilistic Aspects of Triangular Norms, Elsevier, Netherlands, 2005, Pages 189-230.

12. Thangaraj Beaula and B.Johnson, New representation of support of discrete fuzzy number, Bulletin of Mathematics and Statistics Research, 5(3) (2017).

13. W.Voxman, Canonical representation of discrete fuzzy numbers, Fuzzy Sets and Systems, 54 (2001) 457-466.

14. G.Wang, C.Wu, T.A.South and C.Zhao, Representation and operations of discrete fuzzy numbers, Southeast Asian Bulletin of Mathematics, 28 (2005) 1003-1010. 